\title{
Identifying Your Representative Work Based on Credit Allocation
}

\author{
Peng Bao, Jiahui Wang \\ School of Software Engineering, Beijing Jiaotong University \\ CAS Key Lab of Network Data Science and Technology, Institute of Computing Technology \\ \{baopeng,17126211\}@bjtu.edu.cn
}

\begin{abstract}
With the rapid development of scientific impact quantification in the field of science of success, the ability to identify the representative work of a researcher has important implications in a wide range of areas, including hiring, funding, and promotion systems. In this paper, we propose a two-step credit allocation algorithm (TSCA) for identifying the representative work of a researcher. This algorithm explicitly captures the importance of a paper, its relevance to other papers, and the unequally distributed contribution of each citation. We validate TSCA by applying it on the citation data from American Physical Society (APS) in the scenario of identifying the Nobel prize winning papers of the Nobel laureates. Experiments demonstrate that the proposed algorithm can significantly outperform the existing methods.
\end{abstract}

\section{CCS CONCEPTS}

- Information systems $\rightarrow$ Content ranking; • Humancentered computing $\rightarrow$ Reputation systems;

\section{KEYWORDS}

representative work, credit allocation, citation network

\section{ACM Reference Format:}

Peng Bao, Jiahui Wang. 2018. Identifying Your Representative Work Based on Credit Allocation. In $W W W$ '18 Companion: The 2018 Web Conference Companion, April 23-27, 2018, Lyon, France. ACM, New York, NY, USA, 2 pages. https://doi.org/10. $1145 / 3184558.3186901$

\section{INTRODUCTION}

In the last decades, a large number of scientific papers are published online, accelerating the publication of research findings and development of modern science. How to distinguish the scientific impact of a scientist's publications has been an important issue for revealing the quality of academic works [1]. More importantly, identifying the representative work of individual researchers becomes a practical and challenging problem. It is crucial for quantitative assessment of researchers, which affects decisions in a wide range of areas, including hiring, funding, and promotion [2].

This paper is published under the Creative Commons Attribution 4.0 International (CC BY 4.0) license. Authors reserve their rights to disseminate the work on their personal and corporate Web sites with the appropriate attribution.

$W W W$ '18 Companion, April 23-27, 2018, Lyon, France

(C) 2018 IW3C2 (International World Wide Web Conference Committee), published under Creative Commons CC BY 4.0 License.

ACM ISBN 978-1-4503-5640-4/18/04.

https://doi.org/10.1145/3184558.3186901
The most straightforward and widely adopted metric is the citation count. However, it ignores the fact that the importance of each citation is different, which can not objectively reflect the quality of papers. Accordingly, Google's PageRank algorithm was introduced to address this issue, along with its variations $[3,4]$. Despite their initial success in revealing the importance of papers, they are not appropriate for identifying the representative work of individual researcher since the contribution of coauthors in a paper is different $[5,6]$. Recently, in order to incorporate both the importance of a paper and its relevance to other papers, Niu et al. [7] have proposed a self-avoiding preferential diffusion process to rank a scientist's papers. However, they still consider the contribution of each citation equally.

In this paper, we propose a two-step credit allocation algorithm (TSCA) for identifying the representative work of a researcher. Based on the scientific credit allocation mechanism, this algorithm explicitly captures the importance of a paper, its relevance to other papers, and the unequally distributed contribution of each citation, distinguishing itself from the method presented in [7]. We validate TSCA by applying it on the citation data from American Physical Society (APS) in the scenario of identifying the Nobel prize winning papers of the Nobel laureates. Experimental results demonstrate that the proposed algorithm can significantly outperform the state-of-the-art methods.

\section{THE ALGORITHM}

In this paper, we consider the representative work of a researcher as an important paper in his/her field of expertise, which is similar with the definition in [7].

Firstly, we formally express the problem of representative work identification. Suppose we have $M$ authors with the $i$-th author denoted as $a_{i}$ and $N$ papers with the $j$-th paper denoted as $p_{j}$. We denote the author-paper matrix as $\mathbf{A} \in$ $\mathbb{R}^{M \times N}$, with its $(i, j)$-th entry $A_{i j}=1$ if $a_{i}$ is an author of $p_{j}$. Here we denote $\mathbf{a}_{\mathbf{i}}$ as the $i$-th row of matrix $\mathbf{A}$. The paper-paper matrix is denoted as $\mathbf{P} \in \mathbb{R}^{N \times N}$, with its $(i, j)$ th entry $P_{i j}=1$ if $p_{i}$ cites $p_{j}$, and the diagonal entry $P_{i i}=0$. Consequently, the cocitation matrix can be computed as $\mathbf{C}=\mathbf{P P}^{\top}$, where $\mathbf{C} \in \mathbb{R}^{N \times N}$ and its $(i, j)$-th entry $C_{i j}$ denotes the number of papers co-cited by $p_{i}$ and $p_{j}$. Note that we use $d_{i}^{\text {in }}$ and $d_{i}^{\text {out }}$ to denote the number of citations and references of $p_{i}$, respectively.

In the proposed algorithm TSCA, the scores of target author $a_{t}$ 's papers can be expressed as

$$
\mathbf{r}_{\mathbf{t}}=\mathbf{a}_{\mathbf{t}} \mathbf{W}
$$




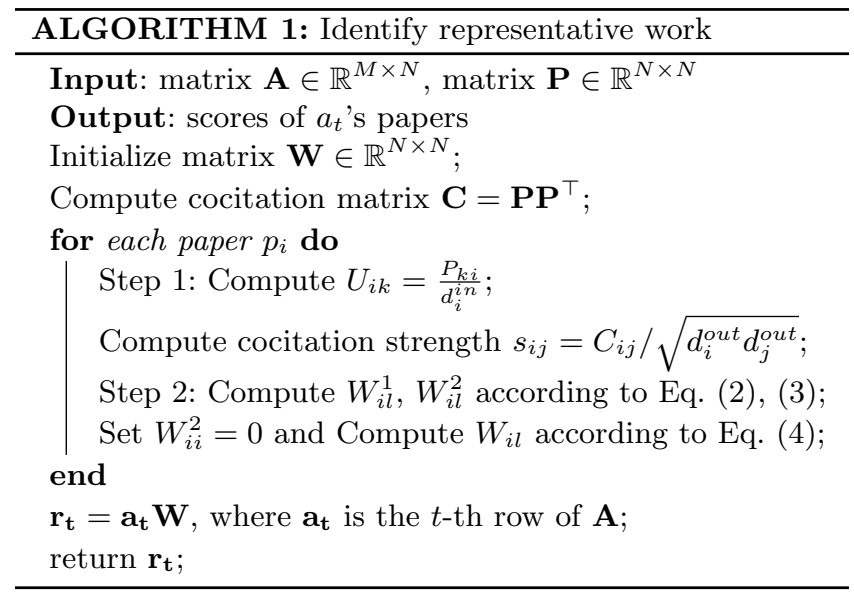

where the matrix $\mathbf{W}$ is the credit matrix with its $(i, j)$-th entry $W_{i j}$ representing the credit value that $p_{j}$ receives from a two-step credit allocation starting from $p_{i}$. The pseudocode is listed in Algorithm 1. The allocation starts with initial credit 1 on paper $p_{i}$. In the first step, the initial credit on $p_{i}$ is evenly distributed to the papers which cite it. Therefore, the credit that $p_{k}$ receives from $p_{i}$ can be computed as $U_{i k}=P_{k i} / d_{i}^{i n}$. In the second step, the credit on paper $p_{k}$ will be unequally allocated back to the papers cited by it based on the cocitation strength, distinguishing from the evenly redistribution method in [7]. For simplicity, we define the cocitation strength $s_{i j}$ between $p_{i}$ and $p_{j}$ as $s_{i j}=C_{i j} / \sqrt{d_{i}^{\text {out }} d_{j}^{\text {out }}}$, which explicitly captures the relevance between two paper and guide us to locate the author's field of expertise.

Given a paper $p_{l}$ which is cited by $p_{k}$, the credit that $p_{l}$ receives in the second step is

$$
W_{i l}^{1}=\sum_{k=1}^{N} s_{i l} \frac{P_{k l} U_{i k}}{d_{k}^{\text {out }}} .
$$

Due to the problem of data sparsity, we further consider the credit assigned from a paper $p_{l^{\prime}}$ which is cited by $p_{k}$ but not written by $a_{t}$. This credit is denoted by $W_{i l}^{2}$ and computed as

$$
W_{i l}^{2}=\sum_{k=1}^{N} \sum_{l^{\prime}=1}^{N} s_{i l} \frac{P_{l^{\prime} l} P_{k l^{\prime}} U_{i k}}{d_{k}^{\text {out }} d_{l^{\prime}}^{\text {out }}} .
$$

Note that we set $W_{i i}^{2}=0$ to ensure that the credit starting from one paper cannot return to itself. In addition, by incorporating the well-known preferential attachment mechanism [1], $W_{i l}$ can be finally expressed by

$$
W_{i l}=d_{l}^{i n}\left(W_{i l}^{1}+W_{i l}^{2}\right) .
$$

\section{EXPERIMENTS}

The dataset used in this paper comprises the papers published in all the journals in APS from 1893 to 2009, consisting of 245,365 authors, 463,344 papers, and 4,692,026 citations. Firstly, we identify the Nobel laureates whose articles published in APS are awarded the Nobel prize. For each laureate, the Nobel winning paper is widely accepted as his/her representative work. In total, we collect 37 laureates along with
Table 1: Prediction performance of four methods.

\begin{tabular}{c|cccc}
\hline Methods & Citation & PageRank & SPD & TSCA \\
\hline \hline mean rank & 3.97 & 3.77 & 3.67 & $\mathbf{3 . 1 0}$ \\
\hline
\end{tabular}

their Nobel prize winning papers, which will be used as our ground truth in the following experiments. As the Nobel prize will significantly attract the attention to the prize winning paper, a more meaningful and much harder problem is to identify the Nobel prize winning paper among the Nobel laureate's papers before he/she receives the prize. Therefore, we focus on ranking each laureate's papers one year before the Nobel prize is received.

In order to quantitatively examine the effectiveness of TSCA, we compute the mean rank of these Nobel prize winning papers in each Nobel laureate's personal ranking list. The smaller the mean rank is, the better the ranking method performs. Three existing methods are selected as baselines: citation count, PageRank [3], and SPD [7].

As shown in Table 1, we find that TSCA exhibits the smallest mean rank among all the methods, with an improvement of $21.9 \%, 17.8 \%$, and $15.5 \%$ compared with citation count, PageRank, and SPD, respectively.

\section{CONCLUSIONS}

In this paper, we propose a two-step credit allocation algorithm for representative work identification. We apply it in the scenario of identifying the Nobel prize winning papers of the Nobel laureates on a APS dataset. Experimental results demonstrate that this algorithm can significantly outperform the existing methods such as citation count, PageRank, and SPD. In the future, time effect will be further investigated.

\section{ACKNOWLEDGMENTS}

The work was supported by the National Natural Science Foundation of China under grant number 61702031, Beijing Excellent Talents Supporting Program under grant number 2017000020124G054, and the Fundamental Research Funds for the Central Universities under grant number 2015RC031. Peng Bao is also funded by CAS Key Lab of Network Data Science and Technology. The authors would also like to thank the anonymous referees for their valuable comments.

\section{REFERENCES}

[1] D. Wang, C. Song, and A.-L. Barabási. Quantifying long-term scientific impact. Science, 342(6154), 127C132, 2013.

[2] R. Sinatra, D. Wang, P. Deville, C. Song, and A.-L. Barabási. Quantifying the evolution of individual scientific impact. Science, 354(6312), 596, 2016.

[3] S. Brin and L. Page. The anatomy of a large-scale hypertextual Web search engine. Computer Networks and ISDN Systems, 30(98), 107-117, 1998.

[4] J. Zhou, A. Zeng, Y. Fan, and Z. Di. Ranking scientific publications with similarity preferential mechanism. Scientometrics, 106, 805816, 2016.

[5] H. W. Shen and A.-L. Barabási. Collective credit allocation in science. Proc. Natl. Acad. Sci., 111(34), 12325C12330, 2014.

[6] P. Bao and C. Zhai. Dynamic credit allocation in scientific literature. Scientometrics, 112, 595-606, 2017.

[7] Q. Niu, J. Zhou, A. Zeng, Y. Fan, and Z. Di. Which publication is your representative work? Journal of Informetrics, 10(8): 842-853, 2016. 